Artigo Original

\title{
Novos modelos alimentares das populações, em prol da segurança alimentar: um panorama da produção científica internacional
}

\author{
New food models for populations meal facing to food safety : an overview on international \\ scientific production
}

\author{
Paula Silva Santos' \\ Thelma Lucchese Cheung" \\ Marcia Maria dos Santos Bortolocci Espejo"II
}

\section{Resumo}

Os sistemas alimentares e as técnicas da produção agropecuária estão diante de desafios complexos, enunciados pelos 17 Objetivos de Desenvolvimento Sustentável da Agenda 2030 (ODS), por exemplo, ao atender a segurança alimentar e nutricional de toda população humana, uma preocupação da Food and Agriculture Organization of the United Nations (FAO), quando a mesma propõe as necessárias transformações nos sistemas agrícolas, nas economias rurais e na forma como são administrados os recursos naturais. Essa ação visa alcançar um mundo livre de fome, desnutrição e qualidade de vida. Neste contexto, esta pesquisa apresenta uma revisão sistemática da bibliografia que trata do tema segurança alimentar, com o objetivo de coletar as principais discussões sobre os novos modelos alimentares das populações. Tratase de uma pesquisa bibliográfica, de ordem descritiva e de abordagem qualitativa. Para o tratamento dos dados coletados, utilizou-se do software Start. As palavras-chave utilizadas para a revisão sistemática foram: "food safety" AND "new food model” AND "food industry”, na base de dados Sciency Direct. Por conta do método de coleta empregado, obtevese 13 artigos internacionais, que discutiam novos modelos alimentares das populações em pró da segurança alimentar. São considerados textos que propiciam novos campos de pesquisa e de avanços das práticas alimentares.

Palavras-chave: 17 Objetivos do desenvolvimento sustentável; Segurança alimentar e nutricional; Cadeia produtiva sustentável; Políticas públicas; FAO

\section{Abstract}

Food systems and agricultural production techniques face complex challenges, as set out in the 17 Sustainable Development Goals (ODS), for example, in addressing the food and nutritional security of the entire human population, a concern of the Food and Agriculture Organization of the United Nations (FAO), when it proposes the necessary changes in agricultural systems, rural economies and the way natural resources are managed. All this action aims to reach a world free of hunger, malnutrition and quality of life. In this context, this research presents a systematic review of the bibliography dealing with food safety, facing to collect the main discussions about the new food models of the populations. It is a purely bibliographical research, descriptive and qualitative approach. For the treatment of the data collected, Star software was used. The keywords described in the sweep of the systematic review were: "food safety" AND "new food model” AND "food industry" in the Sciency Direct database. Due to the method of collection employed, 13 international articles were obtained, which discussed new food models of the populations in favor of food security. They are considered original texts and provide new fields of research and advances in eating practices.

Keywords: 17 Objectives of sustainable development; Food and nutrition security; Sustainable production chain; Public policy; FAO

\footnotetext{
Doutoranda em Administração pelo do Programa de Pós-Graduação em Administração da Escola de Administração e Negócios - PPGAD da ESAN/ UFMS, Universidade Federal de Mato Grosso do Sul - Brasil. E-mail: santos.paulads@gmail.com

" Doutorado em Engenharia de Produção pela Universidade Federal de São Carlos e pela Universidade de Nantes na França, Universidade Federal de Mato Grosso do Sul - Brasil. E-mail:thelma.lucchese@gmail.com

I"' Doutora em Controladoria e Contabilidade pela Universidade de São Paulo -FEA/USP- Brasil. E-mail: marciabortolocci@gmail.com
} 


\section{Introdução}

A alimentação é fator primordial na rotina diária da humanidade, não apenas por ser necessidade básica, mas, principalmente, porque a sua obtenção tornou-se um problema de respeito ao direito humano e de forma complementar de saúde pública, uma vez que o excesso ou falta de hábitos alimentares ou a ausência deles podem causar doenças severas, podendo levar o indivíduo a óbito (ABREU et al, 2001).

De acordo com a Organização para a Cooperação e Desenvolvimento Econômico (OCDE) e com a Organização das Nações Unidas para Agricultura e Alimentação (FAO), será necessário um aumento de $50 \%$ na produção de alimentos até 2050 , devido à previsão estatística de crescimento da população mundial. Ainda de acordo com essas Agências da Organização das Nações Unidas (ONU), o Brasil é o país com maior potencial de contribuição no atendimento dessa demanda adicional de alimentos (OECD; FAO, 2010).

Entretanto, a produção atual de alimentos, provavelmente, não poderá acompanhar o constante aumento da população atual, pois apresenta-se uma situação de muita pressão sobre os escassos recursos naturais inseridos nos complexos ecossistemas, a exemplo do uso da água, das matérias-primas e dos solos agrícolas. Essa pressão é refletida no fato de que, "apesar de um aumento de $30 \%$ na eficiência de recurso, o uso de recursos globais aumentou 50\% nos últimos 30 anos" (THE WORLD WATCH INSTITUTE, 2011, p. 22).

Assim sendo, os sistemas alimentares e as técnicas da produção agropecuária estão diante de desafios complexos, enunciados pelos 17 Objetivos de Desenvolvimento Sustentável da Agenda 2030, quando Organização das Nações Unidas para Agricultura e Alimentação (FAO) adverte que serão necessárias grandes transformações nos sistemas agrícolas, nas economias rurais e na forma como são administrados os recursos naturais, no sentido de se alcançar um mundo livre de fome, de desnutrição e vivência em ambientes socialmente justos (ACSERALD, 2004), o que mais tarde provocou a chamada do paradigma da (In) Justiça Ambiental ou mesmo (In) Justiça Socioambiental (2009).

Dentre as diversas definições sobre Segurança Alimentar e Nutricional (SAN), adota-se o conceito adotado por Maluf (2009, p. 17), mesmo quando a comunidade internacional adota apenas a expressão segurança alimentar. Para o autor, a abrangência conceitual da SAN

é a realização do direito de todos ao acesso regular e permanente a alimentos de qualidade, em quantidade suficiente, sem comprometer o acesso a outras necessidades essenciais, tendo como prática alimentares promotoras de saúde, que respeitem a diversidade cultural e que sejam, social, econômica e ambientalmente sustentáveis.

Antes da abordagem conceitual adotada por Maluf (idem), Castelo Branco (2005, p. 19) já chamava atenção da não dissociação da segurança alimentar dos aspectos nutricionais, quando também estabeleceu seis critérios necessários, inclusive, como método de pesquisa, para a efetivação eficiente da proposta do Programa de Segurança Alimentar e Nutricional (PSAN). Foram eles: 1) disponibilidade de alimentação suficiente; 2) estabilidade do abastecimento de alimentação ao longo do ano e no curso dos anos; 3) acesso à alimentação disponível; 4) distribuição adequada da alimentação na família; 5) boa qualidade de alimentação; e 6) condições de saúda favoráveis à absorção dos alimentos.

De forma complementar, aqui entende-se (In) Justiça Ambiental ou mesmo de (In) Justiça Socioambiental, Acselrad (2009, p. 41) postula que,

nenhum grupo social, seja ele étnico, racial ou de classe, suporte uma parcela desproporcional das consequências ambientais negativas de operações econômicas, decisões de políticas e programas federais, estaduais, locais, assim como da ausência ou omissão de tais políticas [...] asseguram acesso justo e equitativo, direto e indireto, aos recursos ambientais do país.

Como se vê, a reivindicação da Justiça Ambiental pode ser compreendida como o tratamento justo e os envolvimentos plenos dos grupos sociais, independente de sua origem ou renda, nas decisões sobre o acesso, a ocupação e o uso dos recursos ambientais em seus territórios, ou mesmo das rendas e frutos do desenvolvimento econômico que se dá, por conta dos recursos naturais.

Neste sentido, ao se discutir, à luz da ciência, sobre as questões que envolvem a SAN e ou mesmo ao PSAN, concomitantemente, abrange as questões da (In) Justiça Socioambiental, pois ambas estão subordinadas a dois princípios interligados, a saber: o direito humano à alimentação adequada e saudável, não perdendo de vista a soberania alimentar; e o direito humano de se viver num ambiente economicamente viável, ecologicamente equilibrado e socialmente justo.

Neste contexto tem-se a seguinte questão de pesquisa: quais são as principais discussões sobre os novos modelos alimentares das populações, no âmbito da publicação científica internacional, que passam por transformações, em prol da segurança alimentar?

Para responder a esta questão de pesquisa, esta pesquisa tem como objetivo geral fazer uma revisão sistemática bibliográfica sobre o tema segurança alimentar, com o objetivo de averiguar as principais discussões sobre os novos modelos alimentares das populações, em prol da segurança alimentar.

Esta pesquisa é motivada diante do entendimento que ao se discutir sobre segurança alimentar, também leva à reflexão sobre a capacidade de produzir alimentos saudáveis e nutricionalmente desejáveis, possibilidade de entrega para as populações, cujo sistema de produção advém de um ambiente equilibrado e com justiça social aos produtos.

A estrutura textual desta pesquisa inicia-se com esta parte introdutória, com uma breve contextualização da problemática anunciada e o objetivo geral já declarado. Posteriormente, serão explicados os procedimentos metodológicos a serem adotados, para em seguida serem 
apresentados os resultados e análises da revisão sistemática bibliográfica pretendida. Por fim, as Considerações Finais e as Referências que deram suporte a todo relato aqui colocado.

\section{Metodologia}

Esta investigação científica está vinculada ao Grupo de Pesquisa Dinâmica Evolutiva das Organizações Humanas, registrado no Diretório de Pesquisa do Conselho Nacional de Desenvolvimento Científico e Tecnológico (CNPq), do Ministério da Educação (MEC). Para a construção e delineamento desta pesquisa, foi consultado o Code of Conduct and Best Practice Guidelines for Journal Editors (COPE, 2011). Igualmente, acatou as instruções descritas ao longo do Manual Boas Práticas da Publicação Científica, defendidas pela ANPAD (2018).

A abordagem é considerada de pesquisa qualitativa, tendo em vista que ela proporciona ao pesquisador uma melhor análise de realidades, que não pode ser facilmente qualificada (SILVA et al., 2016). Tal abordagem não se utiliza apenas de instrumentos estatísticos, podendo também qualificar ou quantificar os dados coletados por meio de categorias, critérios ou escalas. No caso do presente trabalho, a análise dos dados coletados foi realizada por meio de categorias, utilizando-se das análises de conteúdo (BARDIN, 2004; RICHARDSON, 2007).

Trata-se de uma pesquisa puramente bibliográfica, portanto de fonte secundária, que se utilizou do procedimento de uma revisão sistemática, que de acordo com Galvão; Sawada e Trevizan (2002), advém de um estudo de revisão, que avalia e sintetiza os estudos científicos sobre determinado tema no período selecionado, de forma objetiva e reproduzível, por meio de método científico. Este método de varredura de publicações científicas tem como princípios gerais a exaustão na busca dos estudos analisados, a seleção justificada dos estudos por critérios de inclusão e exclusão explícitos e a analise dos resultados (LIMA; SOARES; BACALTCHUK; 2000).

Para responder a problemática anunciada na parte introdutória e para que o objetivo declarado seja aten- dido, foram realizadas as seguintes etapas. Inicialmente, identificaram-se, as palavras-chaves: "food safety" AND "new food model” AND "food industry". Como já colocado, no Brasil, utiliza-se o termo Segurança Alimentar e Nutricional (SAN), enquanto que no ambiente internacional é apenas mencionada a expressão Segurança Alimentar (food safety). Por esta razão, na processo de varredura dos artigos, usou-se a expressão food safety.

Uma vez definida as palavras chave, utilizou-se a busca pela base de dados do periódico da Capes, vinculado ao "acesso café", estendido à Fundação Universidade Federal do Mato Grosso do Sul (UFMS). Em seguida, realizou-se a busca pela base de dados da Web of Science, no dia 15 de junho de 2018. Na busca, aplicou-se o filtro de pesquisa, restringindo a busca no período de 2013 até 2017, articles and review, publicado no idioma inglês e de acesso livre (open acess).

Após a seleção dos artigos, no qual resultou 85 artigos, foi feito o download no formato bibitex. para que em seguida fosse importado para o software Start (START, 2018), utilizado para a realização da revisão sistemática, de forma digital. Neste software foi analisado o enquadramento dos artigos, o qual seguiu os seguintes critérios: i) resumo disponível e; ii) se os artigos abordavam as três palavras chave "food safety", "new food model" e "food industry".

Seguindo estes critérios, foram coletados, inicialmente, 85 artigos, sendo que destes, 13 continham as três palavras chave, trazendo mais precisão a temática proposta neste assunto. Os demais artigos, traziam outras discussões sobre segurança alimentar, voltada a área química e da saúde. Também teve artigos voltados apenas as discussões das indústrias produtoras de alimentos.

\section{Resultados e Discussões}

Por conta dos procedimentos metodológicos adotados para que o objetivo geral desta pesquisa, já declarado, foi possível coletar os seguintes artigos, tais como mostrados no Quadro 1, que em conjunto evidenciam os modelos para compreensão da segurança alimentar.

Quadro 1

\begin{tabular}{|c|c|c|c|}
\hline Ordem & Título & Autores & Ano \\
\hline 1 & $\begin{array}{l}\text { Identifying baseline food safety training practices for } \\
\text { retail delisusing the Delphi expert consensus method }\end{array}$ & $\begin{array}{l}\text { Kim, Kawon Kathy and O'Bryan, Corliss A. and } \\
\text { Crandall, Phillip G. andRicke, Steven C. and } \\
\text { Neal, Jr., Jack A. }\end{array}$ & 2013 \\
\hline 2 & $\begin{array}{l}\text { Barriers, benefits and motivation factors for the } \\
\text { implementation of foodsafety management system } \\
\text { in the food sector in Harare Province, Zimbabwe }\end{array}$ & $\begin{array}{l}\text { Macheka, Lesley and Manditsera, Faith } \\
\text { Angeline and Ngadze, RuthTambudzai and } \\
\text { Mubaiwa, Juliet and Nyanga, Loveness Kuziwa }\end{array}$ & 2013 \\
\hline 3 & $\begin{array}{l}\text { The new conceptual framework for food supply } \\
\text { chain assessment }\end{array}$ & Manzini, Riccardo and Accorsi, Riccardo & 2013 \\
\hline 4 & $\begin{array}{l}\text { A Split-Sample Revealed and Stated Preference } \\
\text { Demand Model to Examine Homogenous Subgroup } \\
\text { Consumer Behavior Responses to Information and } \\
\text { Food Safety Technology Treatments }\end{array}$ & $\begin{array}{l}\text { Morgan, O. Ashton and Whitehead, John C. } \\
\text { and Huth, William L. andMartin, Greg S. and } \\
\text { Sjolander, Richard }\end{array}$ & 2013 \\
\hline
\end{tabular}


Quadro 1- Conclusão

\begin{tabular}{|c|c|c|c|}
\hline Ordem & Título & Autores & Ano \\
\hline 5 & $\begin{array}{l}\text { Trust makers, breakers and brokers: building trust in } \\
\text { the Australian food system }\end{array}$ & $\begin{array}{l}\text { Wilson, Annabelle and Coveney, John and } \\
\text { Henderson, Julie and Meyer,Samantha and } \\
\text { Calnan, Michael and Caraher, Martin and Webb, } \\
\text { Trevor andElliott, Anthony and Ward, Paul }\end{array}$ & 2013 \\
\hline 6 & Food safety regulatory model in India & Shukla, Seema and Shankar, Ravi and Singh, S. P. & 2014 \\
\hline 7 & $\begin{array}{l}\text { The Production of Safe Food According to Firm Size } \\
\text { and Regulatory Exemption: Application to FSMA }\end{array}$ & Pouliot, Sebastien & 2014 \\
\hline 8 & $\begin{array}{l}\text { Consumers' attitudes, trust and willingness to pay for } \\
\text { food information }\end{array}$ & $\begin{array}{l}\text { Nocella, Giuseppe and Romano, Donato and } \\
\text { Stefani, Gianluca }\end{array}$ & 2014 \\
\hline 9 & $\begin{array}{l}\text { An industry perspective: a new model of food safety } \\
\text { culture excellence and the impact of audit on food } \\
\text { safety standards }\end{array}$ & $\begin{array}{l}\text { Taylor, Joanne and Garat, Jean Pierre and } \\
\text { Simreen, Samer and Sarieddine,Ghida }\end{array}$ & 2015 \\
\hline 10 & $\begin{array}{l}\text { Innovative food quality models - developed as an } \\
\text { interface for modern consumers and sustainable } \\
\text { business }\end{array}$ & $\begin{array}{l}\text { Pamfilie, Rodica and Bobe, Magdalena and } \\
\text { Cristescu, Lavinia and Toma,Maria Alexandra }\end{array}$ & 2016 \\
\hline 11 & $\begin{array}{l}\text { A model for (re)building consumer trust in the food } \\
\text { system }\end{array}$ & $\begin{array}{l}\text { Wilson, Annabelle M. and Withall, Elizabeth } \\
\text { and Coveney, John and Meyer,Samantha B. } \\
\text { and Henderson, Julie and McCullum, Dean and } \\
\text { Webb, Trevor andWard, Paul R. }\end{array}$ & 2016 \\
\hline 12 & $\begin{array}{l}\text { The Role of Auditing, Food Safety, and Food Quality } \\
\text { Standards in the Food Industry: A Review }\end{array}$ & $\begin{array}{l}\text { Kotsanopoulos, Konstantinos } \quad \text { V. } \quad \text { and } \\
\text { Arvanitoyannis, Ioannis S. }\end{array}$ & 2017 \\
\hline 13 & $\begin{array}{l}\text { Contradictions, consequences and the human toll of } \\
\text { food safety culture }\end{array}$ & $\begin{array}{l}\text { Baur, Patrick and Getz, Christy and Sowerwine, } \\
\text { Jennifer }\end{array}$ & 2017 \\
\hline
\end{tabular}

\section{Os modelos para a compreensão da segurança alimentar}

$\mathrm{Na}$ busca realizada para este ensaio, três artigos tratam de modelos que possibilitam a abordagem da segurança alimentar. O trabalho de Wilson et al (2016) apresenta um modelo de melhores práticas, que pode ser utilizado pelos agentes do sistema alimentar para ajudar a (re) construir confiança no sistema alimentar, antes, durante e depois de um incidente alimentar definido como qualquer situação dentro da cadeia de abastecimento onde haja um risco ou potencial risco de doença ou doença confirmada ou lesão associada ao consumo de um alimento ou alimentos.

Estes autores discutem a reconstrução de confiança de um sistema alimentar. O fato ocorre após um acidente de quebra de confiança no sistema alimentar. Tal incidente não foi explicitado no texto. O processo de construção da confiança perpassa por dez itens listados pelos autores “(a) ser transparente, (b) ter protocolos e procedimentos em vigor, (c) ser crível, (d) ser pró-ativo, (e) colocar os consumidores em primeiro lugar, (f) colaborar com as partes interessadas, (g) ser consistente (h) educar as partes interessadas e os consumidores, (i) construir sua reputação e (j) cumprir suas promessas" (WILSON et al 2016, p. 991).

Como estratégia coleta de dados da pesquisa, os autores fizeram um grupo focal com atores envolvidos no sistema de segurança alimentar e, posteriormente, aplicaram 100 questionários, como forma de validação da discussão do grupo focal. Os dez itens foram levantados e discutidos profundamente nos grupos.

A transparência é compreendida pelos autores como o item mais importante, conforme o destaque "os participantes, independentemente do seu papel no sistema alimentar, concordaram que a transparência era a estratégia mais importante para (re) construir a confiança no sistema alimentar após um incidente alimentar” (2016, p. 994). Obstante à construção do conceito de transparência, dá-se pela não clareza dos próprios participantes ao significado do termo.

Transparência significava coisas ligeiramente diferentes para os diferentes atores. Por exemplo, em um contexto de mídia, a transparência consistia em garantir que as fontes de informação fossem citadas, enquanto que, para os atores reguladores e do setor, era mais uma questão de garantir uma boa comunicação e responder às perguntas abertas e honestamente. A transparência também foi discutida em relação a outras estratégias. Por exemplo, os participantes da regulamentação comentaram que a publicação de protocolos e procedimentos para o gerenciamento de incidentes com alimentos é necessária. (2016, p. 994).

Mesmo sem clareza e firmeza no significado entorno do significante transparência, os autores trazem como o principal dos itens e o apresentam como o principal termo do modelo. Como não é o foco deste ensaio destrinchar o artigo estudado, ater-se-á apenas a este significante. Os outros tendem a ser alto explicativo. O modelo que os autores utilizam podem ser verificado na Figura 1, a seguir. 
Figura 1 - Modelo proposto de (re) construção de confiança do consumidor em um sistema alimentar após um incidente

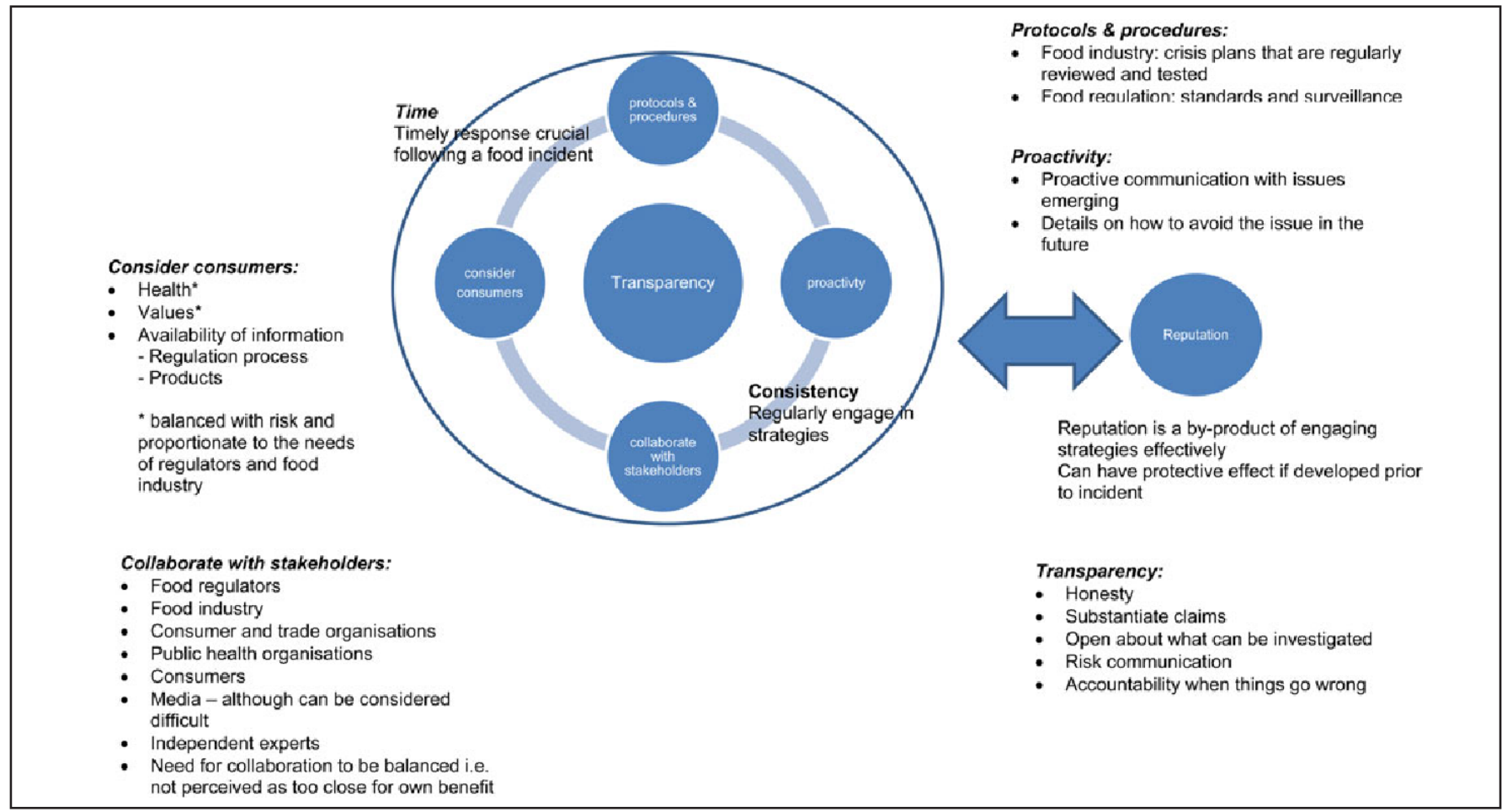

Fonte: Wilson et al (2016)

Como resultado, os autores encontraram as tensões entre atores que estão fora da indústria alimentar, em específico, os jornalistas. Estas tensões tendem a criar um medo injustificado. Contudo, isso pode ser corrigido "através da comunicação e colaboração oportuna e transparente e uma compreensão do papel da mídia na construção do risco, a mídia pode ser utilizada por atores de alimentos de forma eficaz" (2016, p. 998).

O outro modelo de Manzini et al (2013) apresenta um quadro geral e conceitual para a avaliação da cadeia de suprimento de alimentos (FSC) e logística de produtos alimentícios, de acordo com uma visão multidisciplinar e integrada. $\mathrm{O}$ alvo da abordagem integrada proposta para o projeto e gerenciamento da cadeia de suprimentos é o controle simultâneo da qualidade (1), segurança (2), sustentabilidade (3) e eficiência logística (4) de produtos e processos alimentícios ao longo de todo o FSC :

Os mesmos autores utilizam-se de um Estudo de Caso focado no projeto de pacotes, questões de distribuição e apoiado pelo desenvolvimento de um sistema original de controle de loop fechado, como o primeiro passo exemplificativo para uma nova abordagem integrada na avaliação do FSC, de acordo com a estrutura conceitual proposta. Finalmente, o artigo apresenta uma discussão sobre os desafios mais importantes do FSC para a pesquisa pública e privada na indústria e nas instituições acadêmicas.

O trabalho de Taylor et al (2015) apresenta um modelo com quatro categorias. São elas: 1) pessoas; 2) processo; 3) objetivos; e, 4) proatividade. Estas categorias estão subdivididas em 16 dimensões, que são exploradas no artigo. Estas dimensões são complementares e estimulam um processo de melhoria contínua dentre os trabalhadores. Mesmo utilizando alguns significantes diferentes, o modelo se aproxima do modelo de Wilson et al (2016).

\section{Segurança alimentar, qualidade e confiança}

Com as preocupações em relação à segurança alimentar, na qual pautou-se em quantidade e qualidade, os novos modelos de sistemas alimentares vêm discutindo cada vez mais sobre práticas em as indústrias produzam cada vez mais e com maior qualidade, preocupando-se de que forma o consumidor estará consciente de que seus produtos são de qualidade.

Desta forma, tornam-se cada vez mais pertinentes estudos, que discutam sobre a importância da responsabilidade dos produtores em garantir a qualidade dos produtos alimentícios, implementando métodos padronizados de produção e informando os consumidores de maneira correta e completa (Pamfilie et al, 2016).

Ainda de acordo com a autora, a gestão da qualidade dos alimentos representa hoje uma forte garantia de confiança para o consumidor moderno que elimina importantes barreiras com impacto direto no nível de consumo. Wilson et. al (2016) corrobora ao estudar sobre a importância da confiança e como, a confiança do consumidor no suprimento de alimentos é considerada pela mídia, pela indústria de alimentos e pelos governos ao responder às ameaças alimentares.

Esta mesma preocupação está presente no artigo de Macheka et al (2013), que discute a implementação de um sistema de gestão de segurança alimentar no Zimbábue, no Continente Africano. Eles apontam que uma eficaz implementação da gestão da qualidade alimentar trás inúmeros benefícios, entre eles o desenvolvimento pessoal dos funcionários, a melhoria da imagem da empresa, o aumento das vendas e acesso a novos mercados.

A busca por novos mercados é motivador para a implementação de segurança alimentar. Contudo, para 
aumentar a confiança do consumidor, alertam os autores-pesquisadores, é necessário diminuir os riscos da alimentação insegura, ou seja, aquela que não garante qualidade. No viés das indústrias produtoras de alimentos, é necessário preocupar-se com as práticas dos funcionários ao produzir alimentos.

Neste sentido, Kim et. al (2013) trazem preocupações sobre a gravidade do risco de doenças transmitidas por alimentos na indústria de alimentos exóticos, mais ênfase está sendo colocada no treinamento de segurança alimentar para os funcionários da alimentos exóticos. Para eles, torna-se fundamental identificar o treinamento básico mínimo que deve ser dado a cada novo funcionário, a fim de manter o público protegido das doenças transmitidas por alimentos. Estas preocupações estão presentes também na pesquisa de Baptista et al (2016), que analisou o isolamento de uma bactéria para evitar a contaminação, nos alimentos.

Como explorado no modelo de Wilson et al. (2013), há uma necessidade de (re)construção da confiança quando algo errado ocorre. No artigo de Wilson et al (2017) procura abordar como a confiança do consumidor no suprimento de alimentos é considerada pela mídia, pela indústria de alimentos e pelos governos ao responder às ameaças alimentares. O objetivo da pesquisa foi desenvolver modelos de construção de confiança que possam ser implementados, após ameaças alimentares. Os resultados encontrados contribuíram para a literatura sobre a confiança baseada em sistemas e exploraram a confiança como um processo social e regulatório.

O protocolo e os resultados têm sido de interesse e uso para a indústria de alimentos, reguladores de alimentos, grupos de defensores dos consumidores, meios de comunicação que procuram relatar problemas relacionados à alimentação e formuladores de políticas preocupados com o interesse público. Utilizaram-se do estudo de 2013 para a proposição do modelo já apresentado por esta revisão sistemática e publicado em 2017.

Utilizando do arcabouço da Ciência da Psicologia, Nocella et al (2014) analisou a relação das informações sobre os produtos e os consumidores. Apresentou a discussão como uma questão pessoal, remetendo a elementos da psicologia Freudiana, para explicar determinados elementos. Este artigo, juntamente do artigo de Baur et al (2017), que apresentou algumas contradições do sistema de gestão de segurança alimentar foram destoantes dos demais artigos desta revisão. Em busca da garantia da qualidade de produção e fornecimento dos alimentos, fez-se necessário o surgimento de regulamentos que garantissem a segurança alimentar.

\section{Segurança alimentar e regulamentos}

Para Sukla et. al (2014), as mudanças nos padrões de segurança alimentar, exigências sanitárias e fitossanitárias mais rigorosas e melhores normas globais de produtos desencadeiam a necessidade de melhorar a rede legislativa de um país com base no Ponto de Controle Crítico de Análise de Perigos do Codex (HACCP). Devido à multiplicidade de leis, na maioria dos países em desenvol- vimento, a indústria de alimentos processados enfrenta uma grande ameaça à sobrevivência e ao crescimento.

Poliout (2014) corrobora com os resultados do seu estudo, ao apresentar a relação entre a produção da empresa e a segurança alimentar depende de fatores de eficiência específicos da firma e de como a produção e a segurança de alimentos interagem na função de custo. O modelo mostra que, dependendo da distribuição do produto firme e da segurança alimentar, novos regulamentos de segurança alimentar podem aumentar o número de empresas, com ou sem isenção para pequenas empresas. Os regulamentos de segurança alimentar (no caso, a falta de regulamentos firmes) afetam a composição das empresas e podem até mesmo criar entrada por empresas menos eficientes.

O estudo de Kotsanopoulos et al (2017) aponta para a importância dos processos de auditagem para este sistema. Assim, com auditorias haverá melhora na reputação das empresas permitindo o aumento da confiança. Estas melhorias são fundamentais para evitar novos incidentes alimentares.

\section{Considerações Finais}

Esta pesquisa apresentou uma revisão sistemática da bibliografia, sobre o tema Segurança Alimentar e Nutricional, com o objetivo de averiguar as principais discussões sobre os novos modelos alimentares das indústrias em prol da segurança alimentar. As palavras-chave utilizadas para a realização da revisão sistemática foram: "food safety" AND "new food model” AND "food industry", que resultou em 13 artigos.

Os artigos apontaram a importância da discussão de novos modelos alimentares em prol da segurança alimentar, tendo em vista a crescente demanda por alimentos, os quais devem atender os critérios de quantidade e qualidade, além da necessidade de conquistar a confiança do consumidor. Para os pesquisadores brasileiros e para os gestores que têm seus parques industriais construídos, no Brasil, os resultados desta revisão podem ser considerados de grande relevância para auxiliar nas tomadas de decisões empresariais.

Há de se considerar que as discussões que abordagem a Segurança Alimentar está intimamente ligada com a preocupação da Justiça Social, num modelo de produção mais sustentáveis, não somente sobre a ênfase econômica, mas também social e ambiental. Ao contrário, qualquer tipo de sistema produtivo que se afaste da segurança alimentar, da segurança dos alimentos do cuidado dos recursos humanos e ecológicos que estão sobre os complexos ecossistemas, colocarão em risco à saúde do consumo de alimentos e, portanto, da saúde humana e do próprio ecossistema.

Foi compreendido que, estes modelos apresentados são complementares entre si, e como sugestão de pesquisa futura é esmiuçar tais modelos e buscar sua validação empírica. Notou-se também que a regulamentação é uma das maneiras de garantir que a segurança alimentar seja efetivada nas práticas das indústrias alimentares. 


\section{Agradecimentos}

Agradecimentos a revisores, colaboradores e agências de fomento. Estas podem ser colocadas após o artigo ser aprovado para não comprometer a revisão as cegas.

\section{Referências}

ABREU, Simioni Edeli de et al. Alimentação mundial - uma reflexão sobre a história. Saúde e sociedade. v 10(2):3-14, 2001.

ACSELRAD, Henri. Conflitos ambientais no Brasil. Rio de Janeiro: Relume Dumará: Fundação Heinrich Böll, 2004.

ACSELRAD, Henri. O que é Justiça Ambiental. Rio de Janeiro: Garamond, 2009.

ASSOCIAÇÃO NACIONAL DE PÓS-GRADUAÇÃO E PESQUISA EM ADMINISTRAÇÃO (ANPAD). Boas Práticas da Publicação Científica: um manual para autores, revisores, editores e integrantes de Corpos Editoriais. Disponível em: https://www.mackenzie.br/ fileadmin/ARQUIVOS/Public/top/midias_noticias/editora/ old/Editora/Revista_Administracao/Boas_Praticas.pdf. Acesso em: jun. 2018.

BARDIN, Lawrence. Análise do conteúdo. 3. ed. Edições 70, Lda, Lisboa, 2004.

CASTEllo BRANCO, Telma (Org.). Segurança Alimentar e Nutricional no Nordeste do Brasil: Algumas experiências. Rio de Janeiro: Edições Loyola, 2005. (Coleção CERIS, Ano 2, n. 2, 2005).

COPE - Code of Conduct and Best Practice Guidelines for Journal Editors. Code of Conduct, (2011). Disponível em: https://publicationethics.org/files/u7141/1999pdf13. pdf. Acessado em: mar. 2018.

KIM, Kawon Kathy et al. Identifying baseline food safety training practices for retail delis using the Delphi expert consensus method. Food control, v. 32, n. 1, p. 55-62, 2013.

KOTSA N P O U L S, Konstantinos V.; ARVANITOYANNIS, Ioannis S. The Role of Auditing, Food Safety, and Food Quality Standards in the Food Industry: A Review. Comprehensive Reviews in Food Science and Food Safety, v. 16, n. 5, p. 760-775, 2017.

MACHEKA, Lesley et al. Barriers, benefits and motivation factors for the implementation of food safety management system in the food sector in Harare Province, Zimbabwe. Food control, v. 34, n. 1, p. 126-131, 2013.

MALUF, Renato S. Segurança Alimentar e Nutricional. 2. ed. Petrópolis-RJ; Vozes, 2009.
MANZINI, Riccardo; ACCORSI, Riccardo. The new conceptual framework for food supply chain assessment. Journal of food engineering, v. 115, n. 2, p. 251-263, 2013.

MORGAN, O. Ashton et al. A split-sample revealed and stated preference demand model to examine homogenous subgroup consumer behavior responses to information and food safety technology treatments. Environmental and Resource Economics, v. 54, n. 4, p. 593-611, 2013.

NOCELLA, Giuseppe; ROMANO, Donato; STEFANI, Gianluca. Consumers' attitudes, trust and willingness to pay for food information. International journal of consumer studies, v. 38, n. 2, p. 153-165, 2014.

PAMFILIE, Rodica et al. Innovative Food Quality ModelsDeveloped as an Interface for Modern Consumers and Sustainable Business. Amfiteatru Economic Journal, v. 18, n. 43, p. 663-674, 2016.

Patrick; GETZ, Christy; SOWERWINE, Jennifer. Contradictions, consequences and the human toll of food safety culture. Agriculture and Human Values, v. 34, n. 3, p. 713-728, 2017.

POULIOT, Sebastien. The Production of Safe Food According to Firm Size and Regulatory Exemption: Application to FSMA. Agribusiness, v. 30, n. 4, p. 493-512, 2014.

RICHARDSON, Roberto Jarry. Pesquisa social: métodos e técnicas. 3. ed. São Paulo: Atlas, 2007.

SHUKLA, Seema; SHANKAR, Ravi; SINGH, S. P. Food safety regulatory model in India. Food Control, v. 37, p. 401-413, 2014.

SILVA, Eduardo Robini da et al. Caracterização das pesquisas de teses em administração com abordagem qualitativa. Revista de Administração de RoraimaUFRR, Boa Vista, v. 6, n. 1, p. 194-223, jan./jun., 2016.

SOON, Jan Mei; SAGUY, I. Sam. Crowdsourcing: A new conceptual view for food safety and quality. Trends in food science \& technology, v. 66, p. 63-72, 2017.

START, Software. State of the art through systematic review. Disponível em http://lapes.dc.ufscar.br/tools/ start_tool. Acessado em: jan, 2018.

TAYLOR, Joanne et al. An industry perspective: a new model of food safety culture excellence and the impact of audit on food safety standards. Worldwide Hospitality and Tourism Themes, v. 7, n. 1, p. 78-89, 2015.

WILSON, Annabelle et al. Trust makers, breakers and brokers: building trust in the Australian food system. BMC Public Health, v. 13, n. 1, p. 229, 2013. 
WILSON, Annabelle M. et al. A model for (re) building consumer trust in the food system. Health promotion international, v. 32, n. 6, p. 988-1000, 2016.

SAMPAIO. R. F; MANCINI. M.C. Estudos de Revisão Sistemática: Um Guia Para Síntese Criteriosa da Evidência Científica. Rev. bras. fisioter., São Carlos, v. 11, n. 1, p. 83-89, jan./fev. 2007. Disponível em: http:// www.scielo.br/pdf/rbfis/v11n1/12.pdf .Acessado em: 02/ nov/2018.

\section{Contribuição dos autores}

Paula Silva Santos

Contribuições substanciais para a concepção do trabalho; aquisição; análise e interpretação de dados; redigiu o trabalho e realizou uma revisão substancial.

Thelma Lucchese Cheung

Contribuições substanciais para a concepção do trabalho; análise ou interpretação de dados; redigiu o trabalho ou realizou uma revisão substancial.

Marcia Maria dos Santos Bortolocci Espejo

Contribuições substanciais para a concepção do trabalho; análise ou interpretação de dados; redigiu o trabalho ou realizou uma revisão substancial. 